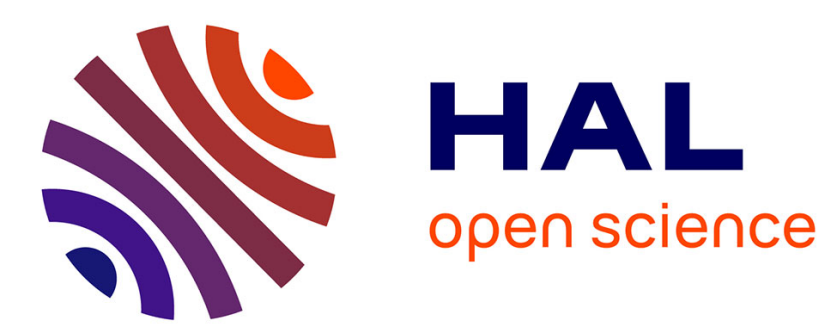

\title{
A conceptually improved TD-DFT approach for predicting the maximum absorption wavelength of cyanine dyes
}

Kamel Meguellati, Sylvain Ladame, Martin Spichty

\section{- To cite this version:}

Kamel Meguellati, Sylvain Ladame, Martin Spichty. A conceptually improved TD-DFT approach for predicting the maximum absorption wavelength of cyanine dyes. Dyes and Pigments, 2011, 90 (2), pp.114-118. 10.1016/j.dyepig.2010.12.001 . hal-02929373

\section{HAL Id: hal-02929373 \\ https://hal.science/hal-02929373}

Submitted on 17 Nov 2021

HAL is a multi-disciplinary open access archive for the deposit and dissemination of scientific research documents, whether they are published or not. The documents may come from teaching and research institutions in France or abroad, or from public or private research centers.
L'archive ouverte pluridisciplinaire HAL, est destinée au dépôt et à la diffusion de documents scientifiques de niveau recherche, publiés ou non, émanant des établissements d'enseignement et de recherche français ou étrangers, des laboratoires publics ou privés. 


\title{
A conceptually improved TD-DFT approach for predicting the maximum absorption wavelength of cyanine dyes
}

\author{
Kamel Meguellati $\$$, Sylvain Ladame $\$$, and Martin Spichty $\$, \S, *$ \\ \$Institut de Science et d'Ingénierie Supramoléculaires, Université de Strasbourg, 8 allée Gaspard \\ Monge, B.P. 70028, 67083 Strasbourg Cedex; \\ ${ }^{\S}$ Current address: Laboratoire de Biologie Moléculaire de la Cellule, Différenciation et Cycle Cellulaire, \\ Ecole Normale Supérieure de Lyon, 46 allée d'Italie, 69364 Lyon Cedex 07. \\ *To whom correspondence should be addressed, Email: martin.spichty@,ens-lyon.fr.
}


ABSTRACT. Cyanine dyes have found valuable applications in modern bioresearch because of their biocompatibility, high molar absorptivity and moderate fluorescence quantum yield. Of particular interest for sensing and labeling applications is the fact they can cover a very large spectral range (from blue to Infra-Red). To design and select the most appropriate dyes for a given application the computational prediction of the absorption wavelength (prior to the costly chemical synthesis) serves as a valuable guidance. However, predicting absorption and emission wavelengths of such compounds remains a challenging task. Herein, we report a fast and highly accurate computational approach which allows the prediction of the maximum absorption wavelength for a wide range of cyanine dyes, including symmetrical and unsymmetrical, trimethine and pentamethine cyanine dyes but also unusual imino-based analogues. In addition to the vertical excitation energy (calculated from time-dependent density functional theory), the approach makes use of a novel correction term that is based on the ground-state zero-point vibrational energy (ZPVE). The correction term is statistically significant $(F-$ test), and it reduces the average error and maximal error of the prediction by a factor of two. We anticipate that the concept of including the ZPVE into the calculation of the maximum absorption wavelength can be used also for other families of dyes to improve their predictability.

KEYWORDS: quantum chemical calculations, time-dependent density functional theory, linear scaling approach, zero-point vibrational energy, empirical correction. 


\section{Introduction}

Dyes are molecules that display light absorption in the long-wavelength region and thereby give raise to color perception [1]. Of particular interest in modern bioresearch are dyes capable of emitting a fluorescent signal upon excitation with a single (or multiple) photon(s). Fluorescence arises when the relaxation corresponding to the transition of an electron from the first singlet excited state $\left(\mathrm{S}_{1}\right)$ to the singlet electronic ground state $\left(\mathrm{S}_{0}\right)$ occurs via photon emission. Since the discovery of the first natural fluorophore in 1845 [2], numerous fluorescent small molecules have been engineered that cover a very broad spectral range (from blue to infra-red) [3]. Among them, cyanine dyes have found valuable applications (e.g. for optical data storage, proteomic, labeling of biomolecules) [4]. They generically consist of a conjugated system based on a polymethine chain linking two nitrogen-containing heterocycles (e.g. indoles, benzothiazoles) [5].

\section{(Insert Figure 1 here)}

We have recently introduced a new class of dyes differing from the well known cyanine dyes by one or two $\mathrm{C} \rightarrow \mathrm{N}$ substitutions within the polymethine chain (Figure 1) [6,7]. While the potential of polymethine cyanine dyes for labeling purposes is now well-established [8], the possible use of their imino analogues for either sensing malondialdehyde (MDA) or alkylating DNA/RNA nucleobases has also been recently demonstrated [7].

Depending on the process or metabolite that needs to be sensed, fluorescent molecules absorbing and emitting in specific regions of the spectrum are necessary. Therefore, understanding and tuning the spectroscopic properties of cyanine dyes remains of vital interest. The prediction of spectroscopic properties from numerical calculations provides valuable input in this regard; e.g., the computational 
appraisal of the absorption wavelength prior to the costly chemical synthesis may serve as guidance for the design and selection of the most appropriate dyes for a given application.

Absorption wavelengths of dyes have been calculated by various approaches ranging from simplistic $\pi$-electron models and semi-empirical all-valence-electron methods to computationally intensive $a b$ initio model chemistries (for a concise overview, see reference [9]). In recent years time-dependent density functional theory (TD-DFT)[10] has increasingly been used [9, 11-20] to fill the gap between the semi-empirical and high-level ab initio methods, i.e., TD-DFT is a first-principles method that overcomes short-comings of the former methods (e.g., electron correlation) and yet it is still applicable to much larger systems (about 100 second-row atoms) than the latter [9].

Despite considerable efforts in TD-DFT, the accurate prediction of maximum absorption wavelengths from computations remains, however, a challenging task. There are several reasons for this. Beside the Hamiltonian approximations (e.g., exchange correlation functional, basis set, treatment of solvent) the calculations usually differ also conceptually from the experiment, i.e., a discrete value for the vertical excitation energy of a ground-state minimum-energy structure is calculated $\left(\Delta E_{\mathrm{v}}\right)$, whereas the reported value of the experiment corresponds to the energy value of the absorption maximum $\left(\Delta E_{\max }\right)$ which depends on the virbonic structure of the dye; see also discussion by Champagne et al. [14].

Herein, we report the development of an optimized approach for predicting with high accuracy the maximum absorption wavelength of polymethine cyanine dyes as well as that of their imino analogues. As a first test, we use a linear scaling approach[14] that estimates the maximum absorption wavelength $\Delta E_{\max }$ solely from the TD-DFT-calculated $\Delta E_{\mathrm{v}}$. We then propose a conceptual refinement of the standard approach by introducing an empirical correction term that is based on the zero-point vibrational energy. The statistical significance of the ZPVE-based correction is verified ( $F$-test), and the portability of this methodological development to other families of dyes is discussed. 


\section{Material and methods}

Compounds 1-14 were synthesized following experimental procedures previously reported by us [6,7] and others [21]. Ultraviolet absorption spectra of analytically pure samples were recorded on a V-670 UV-Visible spectrophotometer from JASCO in a $10 \mathrm{~mm}$ pathlength cuvette.

\section{Theory and calculations}

We build on a linear scaling approach [14]: $\Delta E_{\max }$, is obtained from the TD-DFT-based $\Delta E_{\mathrm{v}}$ through an empirical equation:

$$
\Delta E_{\max }=\alpha \Delta E_{\mathrm{v}}+\beta
$$

where $\alpha$ and $\beta$ are two parameters that are fitted from a series of dyes with known experimental maximum absorption wavelengths. We used the cyanine dyes of Table 1 for which $\Delta E_{\max }$ has been measured under the same experimental conditions, i.e., same solvent (DMSO), counter ion, and temperature $[6,7]$.

Variants of Eq. 1 are known where additional terms for solvent effects are included [15,16], or where the absorption wavelengths of maximal intensity is calculated based on multiple QSAR descriptors [22]. Here we used the following extension of Eq. 1:

$$
\Delta E_{\max }=\alpha \Delta E_{\mathrm{V}}+\beta+\gamma\left(\mathrm{ZPVE} / n_{\mathrm{DOF}}-\delta\right)^{2},
$$

with ZPVE the zero-point vibrational energy (in the harmonic approximation) [23], $n_{\text {DOF }}$ the number of vibrational degrees of freedom ( $3 N-6$ for non-linear molecules, with $N$ the number of atoms), and $\gamma, \delta$ are two additional parameters to be fitted. 
Several methods of calculating $\Delta E_{\mathrm{v}}$ were tested to fit Eq.1, the "best" method (highest $R^{2}$ and $Q^{2}$, see below) was then used to fit Eq. 2. The minimum-energy structure was calculated on the B3LYP/6-31G* level of theory either in the gas phase or in solution (using the PCM solvation model [24]); in both cases a tight minimization was performed and the localization of the stationary points was verified with a frequency calculation that yielded ZPVE. Two different functionals, B3LYP [25,26] and PBE0 [27] (also named PBE1PBE [28]), in combination with three different basis sets, 6-31G*, 6-311G*, and 6$311+\mathrm{G}^{* *}$, were then used to determine the vertical excitation energy $\Delta E_{\mathrm{v}}$ by a single-point TD-DFT calculation (with and without solvation model) [29]. All calculations were carried out with the program Gaussian09 [28].

For each prediction model we calculated the squared correlation coefficient, $R^{2}$, between the calculated and experimental $\Delta E_{\max }$ values, as well as the leave-one-out cross-validated $R^{2}$, named $Q^{2}$; the former parameter is a measure of the goodness-of-fit, the latter of the goodness-of-prediction [30]. When two models are compared that are based on different equations (with a different number of parameters, such as Eq. 1, Eq. 2 and variants of the latter; see section Results and Discussion), an increase in $R^{2}$ when going from the smaller, nested equation to the larger equation does not always indicate a statistically significant improvement of the model. We therefore performed a likelihood-ratio test (F-test) [31]. Under the null hypothesis that the larger model does not fit the data better than the smaller, nested model, the value

$$
F=\left[\left(R_{1}^{2}-R_{\mathrm{s}}^{2}\right) /\left(1-R_{1}^{2}\right)\right] \times\left[\left(p_{1}-p_{\mathrm{s}}\right) /\left(N-p_{1}\right)\right],
$$

will have a $F$-distribution with $p_{1}-p_{\mathrm{s}}$. numerator and $N-p_{1}$ denominator degrees of freedom. $R_{1}^{2}$ is the correlation coefficient for the model with the larger number of parameters $\left(p_{1}\right) ; R_{\mathrm{s}}{ }^{2}$ and $p_{\mathrm{s}}$ are the respective quantities of model with the simpler, nested equation. $N$ is the number of data points available for the fit. To reject the null hypothesis, the value of Eq. 3 needs to be greater than the value of 
the $F$-distribution for some desired false-rejection probability ( $P$ value). For the comparison of the fits with Eq. 2 and Eq. 1, for example, the value of the $F$-distribution (two numerator and ten denominator degrees of freedom) is 4.10 for the critical $P$ value of 0.05 .

\section{(Insert Table 1 here)}

\section{Results and discussion}

It has been noted previously that the use of a solvation model can improve significantly the agreement between experimental $\Delta E_{\max }$ and predicted values from Eq. 1 [14]. When a pure gas phase method, i.e., both geometry optimization and single-point calculation are carried out in gas phase (see values in parentheses of Table 2), is compared with a method where the latter step is carried out with the PCM solvation model, the correlation coefficient $R^{2}$ and the leave-one-out cross-validated correlation coefficient $Q^{2}$ increase by about $20 \%$. When both steps are carried out in solution, $R^{2}$ and $Q^{2}$ further increase; the effect is, however, smaller. Improvement is also possible when the functional B3LYP is replaced by PBE0 [15]. Furthermore, increasing the basis set from 6-31G* to 6-311G* increases $R^{2}$ and

$Q^{2}$, but $6-311 \mathrm{G}^{*}$ and $6-311++\mathrm{G}^{* *}$ yield essentially identical results. The highest goodness-of-fit and goodness-of-prediction are obtained for the method PBE0/6-311++G**(PCM)//B3LYP/6-31G*(PCM) (Figure 2a). With this model the error in the prediction of $\Delta E_{\max }$ ranges from -0.07 to $+0.12 \mathrm{eV}$ ( -28 to $+21 \mathrm{~nm})$, the root-means-square error is $0.06 \mathrm{eV}(15 \mathrm{~nm})$.

\section{(Insert Table 2 \& Figure 2 here)}

The quality of the prediction with Eq. 1 (correlation coefficient $R^{2}=0.968$, maximal error $=0.12 \mathrm{eV}$ ) is notably worse than previously reported for analogous polymethine cyanine dyes $\left(R^{2}=0.988\right.$, maximal error $=0.04 \mathrm{eV}$ ) [14]. In this study, however, we used a much more heterogeneous data set for the fitting of Eq. 1 including mono- and di-imino dyes. Especially di-imino derivatives show large 
deviations between prediction and experiment as indicated by the arrows in Figure 2a. Thus for this heterogeneous set the simple linear scaling approach of Eq. 1 fails to reproduce the experimental order of the $\Delta E_{\max }$ values.

We note significant differences in the zero-point vibrational energy (ZPVE) among the studied dyes. The total ZPVE depends strongly on the number of atoms, so that its comparison between molecules with different number of atoms is not very informative. Normalization (i.e., division) by the number of degrees of freedom $\left(n_{\mathrm{DOF}}\right)$ allows, however, the identification of changes in the distribution of normal mode frequencies. Such changes may influence the "sharp" vibrational coupling of cyanine dyes with the solvent [32]; thereby alternate the strength of the solute-solvent interaction (e.g., change the solvation contribution to the free energy values in the Marcus model [33]) and slightly shift the absorption maximum. The vertical excitation energy $\Delta E_{\mathrm{v}}$ (calculated from an energy-minimized structure with an implicit solvation model) does not capture such solvent coupling effects.

\section{(Insert Figure 3 here)}

The ZPVE is also of relevance in terms of vibronic contributions (when calculating $\Delta E_{\max }$ from $\Delta E_{\mathrm{v}}$ ). For cyanine dyes the band with the highest intensity corresponds to a transition between the vibrational ground states of $\mathrm{S}_{0}\left(\mathrm{v}^{\prime \prime}=0\right)$ and $\mathrm{S}_{1}\left(\mathrm{v}^{\prime}=0\right)$ [34]. Consider for the moment a $(0,0)$-transition (Figure 3$)$ of a hypothetical molecular model system with $n_{\mathrm{DOF}}$ identical vibrations of frequency $v$ and ZPVE $=1 / 2 n_{\mathrm{DOF}}$ $h v$. The frequency does not change upon excitation, but the equilibrium geometry of the excited state, $r_{\mathrm{e}}$, differs slightly from that of the ground-state, $r_{\mathrm{e}}{ }^{\prime \prime}$, where the difference in reduced (dimensionless) coordinates is denoted by $d$. Since it is $\Delta E_{\max }=D_{\mathrm{o}}$ (see Figure 3) we find $\Delta E_{\max }=\Delta E_{\mathrm{v}}-n_{\mathrm{DOF}} 1 / 2 h v d^{2}=$ $\Delta E_{\mathrm{v}}-$ ZPVE $d^{2}$, where the last term, ZPVE $d^{2}$, is the vibrational reorganization energy [33]. For systems with large $n_{\mathrm{DOF}}$ the value $d$ should be smaller than for systems with small $n_{\text {DOF }}$ because for large molecules the relative geometric change per degree of freedom is smaller upon excitation than for small 
molecules. If we assume that the total geometric change in reduced coordinates is constant among dyes of the same family (i.e., $n_{\mathrm{DOF}} d^{2}=\mathrm{const}$ ), we have

$$
\Delta E_{\max }-\Delta E_{\mathrm{v}} \propto \mathrm{ZPVE} / n_{\mathrm{DOF}}
$$

i.e., the difference between the maximum absorption energy and the vertical excitation energy is proportional to $\mathrm{ZPVE} / n_{\mathrm{DOF}}$. Eq. 4 is, of course, a drastically oversimplified representation for realistic molecular systems. But it may reflect to some extend relevant information when comparing different members of dyes from the same family (e.g., cyanine dyes) relative to each other. Using the calculated ZPVE values of the studied dyes the hypothetical frequency $v=(2 / h) \mathrm{ZPVE} / n_{\text {DOF }}$ ranges between 1180 and $1273 \mathrm{~cm}^{-1}$. Interestingly this is within the typical frequency span of vibrations that get excited upon $\mathrm{S}_{0} \rightarrow \mathrm{S}_{1}$ transitions in cyanine dyes $\left(1200 \pm 200 \mathrm{~cm}^{-1}[35]\right)$.

We tried to include possible solvent coupling effects and vibronic contributions into the calculation of $\Delta E_{\max }$ by complementing Eq. 1 with an empirical $\mathrm{ZPVE} / n_{\mathrm{DOF}}$-based correction term (Eq. 2). The improvement of the prediction with Eq. 2 in comparison to Eq. 1 is obvious (Figure 2 b). $R^{2}$ and $Q^{2}$ increase from 0.968 to 0.994 and from 0.956 to 0.989 , respectively. The error in the prediction of $\Delta E_{\max }$ ranges now from -0.04 to $+0.06 \mathrm{eV}(-10$ to $+12 \mathrm{~nm})$, the root-mean-square error is $0.03 \mathrm{eV}(7 \mathrm{~nm})$. Thus, both the maximal and root-mean-square error drop by a factor of two in comparison with the best model of Eq. 1. The model based on Eq. 2 yields also the correct order of $\Delta E_{\max }$ values for the set of studied cyanine dyes; an exception is the couple $\mathbf{8}$ and $\mathbf{9}$ for which the experimental $\Delta E_{\max }$ differs by less than $0.02 \mathrm{eV}$ (i.e., the difference is smaller than the root-mean-square error of the model).

When we compare the models of Eq. 2 and Eq. 1 in a likelihood-ratio test under the null hypothesis that Eq. 2 does not fit the data better than Eq. 1, we find an $F$ value of 21.3. This is much larger than the value of the $F$-distribution (4.10) for a critical false-rejection probability of 0.05 . In fact, the null hypothesis can be rejected with a certainty of $99.97 \%\left(P\right.$ value $\left.=2.48 \times 10^{-4}\right)$. This underlines the statistical significance of the correction term in Eq. 2.

We tested also a linear form of the correction term $\left(\gamma\left[\mathrm{ZPVE} / n_{\mathrm{DOF}}\right]\right.$ with only one additional parameter 
to fit) and a cubic form $\left(\gamma\left[\mathrm{ZPVE} / n_{\mathrm{DOF}}\right]+\delta\left[\mathrm{ZPVE} / n_{\mathrm{DOF}}\right]^{2}+\varepsilon\left[\mathrm{ZPVE} / n_{\mathrm{DOF}}\right]^{3}\right.$, i.e., a third-order polynomial with three additional parameters). The former fits the data notably worse $\left(R^{2}=0.985\right.$ and $Q^{2}$ $=0.978)$ than the quadratic form (Eq. 2), the latter fits marginally better $\left(R^{2}=0.995, Q^{2}=0.989\right)$. When comparing the models of the quadratic correction term and the nested, linear term in a likelihood-ratio test, we find an $F$ value of 14.3 which corresponds to a $P$ value of 0.004 (one numerator and ten denominator degrees of freedom). The null hypothesis (i.e., the quadratic correction term does not fit the data better than the linear correction term) can be rejected with a certainty of $99.6 \%$. The comparison of the models of the cubic correction term and the nested, quadratic term, yields, however, an $F$ value of only 1.2 which corresponds to a $P$ value of 0.302 (one numerator and nine denominator degrees of freedom). This is six times larger than the critical false-rejection probability of 0.05 . The quadratic form of the correction term is therefore sufficient to describe the dependence of $\Delta E_{\max }$ on $\mathrm{ZPVE} / n_{\mathrm{DOF}}$.

\section{Conclusions}

A more accurate TD-DFT prediction of the maximum absorption wavelength of symmetrical or unsymmetrical cyanine dyes is possible by introducing a correction term that potentially accounts for vibronic contributions and solvent coupling effects. Interestingly, with this correction term the prediction is also successful for "unusual" cyanine dyes that contain one or two imino linkage(s) within their polymethine chain. The maximal error in the prediction is $12 \mathrm{~nm}$ with correction term, instead of $28 \mathrm{~nm}$ without correction term; the improvement of the agreement (between prediction and experiment) is statistically significant. The correction term is based on the zero-point vibrational energy (ZPVE) normalized by the number of vibrational degrees of freedom. With the aid of a simplified model system it was shown that the correction term possibly accounts for the conceptual difference between the maximum absorption energy (as obtained from experiments, see $\Delta E_{\max }$ in Figure 3) and the vertical excitation energy (as obtained from quantum chemical calculations, $\Delta E_{\mathrm{v}}$ ). The model is not limited to cyanine dyes but it is generally applicable to dyes with a dominant $(0,0)$-transition. For dyes with larger 
geometric changes upon excitation (i.e., for dyes where the most favorable transition leads to a vibrationally excited $\mathrm{S}_{1}$.state) the difference between $\Delta E_{\max }$ and $\Delta E_{\mathrm{v}}$ also depends on ZPVE: the energy for the most probable transition corresponds to the zero-point corrected vertical transition energy (Franck-Condon principle); the normalization of ZPVE by $3 N-6$ could be less important though. We anticipate therefore that ZVPE-based correction terms can be used also for many families of dyes to improve the prediction of their maximum absorption wavelengths. The investigation of the theoretical basis of the correction term, especially its quadratic nature (e.g., anharmonicity effects) is subject to future studies.

Acknowledgement. We thank Prof. Martin Karplus for valuable discussions, and GENCI (Grand Equipement National de Calcul Intensif, France) for computational resources. MS thanks ISIS and the University of Strasbourg for hosting him as guest assistant professor. SL and KM are grateful for financial support from Centre National de la Recherche Scientifique (CNRS) and the International Center for Frontier Research in Chemistry (FRC).

Supplementary data. Chemical structures of compounds 1-14.

[1] Lotto RB, Purves D. The empirical basis of color perception. Conscious Cogn 2002; 11: 609-29.

[2] Herschel JFW. On a case of superficial colour presented by a homogeneous liquid internally colourless. Phil Trans R Soc1845; 135: 143-5.

[3] Lavis LD, Raines RT. Bright ideas for chemical biology. ACS Chem Biol 2008; 3: 142-55.

[4] Mishra A, Behera RK, Behera PK, Mishra BK, Behera GB. Cyanines during the 1990s: a review.. Chem Rev 2000; 100: 1973-2011.

[5] Benson RC, Kues HA. Absorption and fluorescence properties of cyanine dyes. J Chem Engin Data $1977 ; 22: 379-83$. 
[6] Meguellati K, Spichty M, Ladame S. Reversible Synthesis and Characterization of Dynamic Imino Analogues of Trimethine and Pentamethine Cyanine Dyes. Org Lett 2009; 11: 1123-6.

[7] Meguellati K, Spichty M, Ladame S. Synthesis, spectroscopic and DNA alkylating properties of malondialdehyde (MDA) bis-imine fluorescent adducts. Mol BioSyst 2010; 6. 1694-9.

[8] Gonçalves MS. Fluorescent Labeling of Biomolecules with Organic Probes. Chem Rev 2009; 109: 190-212.

[9] Fabian J. TDDFT-calculations of Vis/NIR absorbing compounds. Dyes Pigm 2010; 84: 36-53.

[10] Burke K, Werschnik J, Gross EKU. Time-dependent density functional theory: Past, present, and future. J Chem Phys 2005; 123: 062206.

[11] Bamgbelu A, Wang J, Leszczynski J. TDDFT Study of the Optical Properties of Cy5 and Its Derivatives. J Phys Chem A 2010; 114: 3551-5.

[12] Jacquemin D, Wathelet V, Perpète EA, Adamo C. Extensive TD-DFT Benchmark: Singlet-Excited States of Organic Molecules. J Chem Theory Comput 2009; 5: 2420-35.

[13] Hagberg DP, Marinado T, Karlsson KM, Nonomura K, Qin P, Boschloo G, Brinck T, Hagfeldt A, Sun L. Tuning the HOMO and LUMO Energy Levels of Organic Chromophores for Dye Sensitized Solar Cells. J Org Chem 2007; 72: 9550-6.

[14] Champagne B, Guillaume M, Zutterman F. TDDFT investigation of the optical properties of cyanine dyes. Chem Phys Lett 2006; 425: 105-9.

[15] Wang LY, Chen QW, Zhai GH, Wen ZY, Zhang ZX. Investigation of the structures and absorption spectra for some hemicyanine dyes with pyridine nucleus by TD-DFT/PCM approach. J Mol Struct (Theochem) 2006; 778: 15-20.

[16] Fu YL, Huang W, Lia CL, Wang LY, Wei YS, Huang Y, Zhang XH, Wen ZY, Zhan ZX. 
Monomethine cyanine dyes with an indole nucleus: Microwave-assisted solvent-free synthesis, spectral properties and theoretical studies. Dyes Pigm 2009; 82: 409-15.

[17] Dierksen M, Grimme S. The Vibronic Structure of Electronic Absorption Spectra of Large Molecules: A Time-Dependent Density Functional Study on the Influence of "Exact" Hartree-Fock Exchange. J Phys Chem A 2004; 108: 10225-37.

[18] Luňák Jr. S, Vyňuchala J, Valac M, Havel L, Hrdina R. The synthesis, absorption and fluorescence of polar diketo-pyrrolo-pyrroles. Dyes Pigm 2009; 82: 102-8.

[19] Guillaumont D, Nakamura S. Calculation of the absorption wavelength of dyes using timedependent density-functional theory (TD-DFT). Dyes Pigm 2000; 46: 85-92.

[20] Guillaume M, Liégeois V, Champagne B, Zutterman F. Time-dependent density functional theory investigation of the absorption and emission spectra of a cyanine dye. Chem Phys Lett 2007; 446: 165 9.

[21] Hamer FM. The Cyanine dyes and related compounds. Interscience Publishers: New York, London; 1964.

[22] Fayet G, Jacquemin D, Wathelet V, Perpete EA, Rotureau P, Adamo C. Excited-state properties from ground-state DFT descriptors: A QSPR approach for dyes . J Mol Graph Modell 2010; 28: 465-71.

[23] McQuarrie DA, Simon JD. Molecular Thermodynamics. University Science Books: Sausalito; 1999.

[24] Tomasi J, Mennucci B, Cancès E. The IEF version of the PCM solvation method: An overview of a new method addressed to study molecular solutes at the QM ab initio level. J Mol Struct (Theochem) 1999; 464: 211-26.

[25] Becke AD. new mixing of Hartree-Fock and local density-functional theories. J Chem Phys 1993; 
98: $1372-7$.

[26] Lee C, Yang W, Parr RG. Development of the Colle-Salvetti correlation-energy formula into a functional of the electron density. Phys Rev B 1988; 37: 785-9.

[27] Adamo C, Barone V. Toward reliable density functional methods without adjustable parameters: The PBE0 model. J Chem Phys 1999; 110: 6158-69.

[28] Frisch M. et al. Gaussian 09 Revision A.02. Gaussian, Inc.: Wallingford; 2009.

[29] Stratmann RE, Scuseria GE, Frisch MJ. An efficient implementation of time-dependent densityfunctional theory for the calculation of excitation energies of large molecules. J Chem Phys 1998; 109: 8218-24.

[30] Leach AR, Gillet VJ. An introduction to Chemoinformatics. Revised Edition. Springer: Dordrecht; 2007.

[31] Allen MP. Understanding Regression Analysis. Plenum Press: New York; 1997.

[32] Sheppard SE, Brigham HR. Some Effects of Solvents upon the Absorption Spectra of Dyes. III. Temperature and Organic Solutions of Cyanine Dyes. J Am Chem Soc 1944; 66: 380-4.

[33] Marcus RA. Relation between charge transfer absorption and fluorescence spectra and the inverted region. J Phys Chem 1989; 93: 3078-86.

[34] Mustroph H, Reiner K, Mistol J, Ernst S, Keil D, Hennig L. Relationship between the molecular structure of cyanine dyes and the vibrational fine structure of their electronic absorption spectra. ChemPhysChem 2009; 10: 835-40.

[35] Pouradier J. Remarque sur le spectre d'absorption des cyanines en solution. J Chim Phys 1964; 61: 1107-14. 


\section{Figure Legends.}

Figure 1. General structure of polymethine $(X=C H)$ and polyimine $(X=N)$ cyanine dyes covering a spectral range from blue to red. Pictures of selected dyes in solution (DMSO) are also given for illustration.

Figure 2. Plot of calculated and experimental $\Delta E_{\max }$ values for cyanine dyes of Table 1 using Eq. 1 (a) and Eq. 2 (b). The fitted parameters are $\alpha=1.2104, \beta=-0.8334 \mathrm{eV}$ for Eq. 1 , and $\alpha=1.1985, \beta=-$ $0.8687 \mathrm{eV}, \gamma=1.2771 \times 10^{4}(\mathrm{eV})^{-2}$, and $\delta=7.7045 \times 10^{-2} \mathrm{eV}$, for Eq. 2, respectively. The value of $\delta$ is close to the value of ZPVE $/ n_{\mathrm{DOF}}$ averaged over all dyes $\left(<\mathrm{ZPVE} / n_{\mathrm{DOF}}>=7.6113 \times 10^{-2} \mathrm{eV}\right)$. The arrows in a) indicate di-imino derivates with large deviations between experiment and prediction using Eq. 1.

Figure 3. Schematic representation of the potential energy surfaces of the ground-state, $\mathrm{S}_{0}$, and the first excited state, $\mathrm{S}_{1}$. The value $\Delta E_{\mathrm{v}}$ (thin arrow) corresponds to the potential energy difference between $\mathrm{S}_{1}$ and $\mathrm{S}_{0}$ at the equilibrium geometry of the ground state $\left(r_{\mathrm{eq}}{ }^{\prime \prime}\right)$. Note that $\Delta E_{\mathrm{v}}$ is a purely theoretical quantity (which has no experimental equivalence); it corresponds to the vertical electronic transition energy traditionally calculated in quantum-chemical calculations, e.g., single-point TD-DFT calculations. For fluorescent molecules such as cyanine dyes the equilibrium geometry of $\mathrm{S}_{1}\left(r_{\mathrm{eq}}\right)$ differs only slightly from that of $\mathrm{S}_{0}[34]$, so that the most intensive transition occurs between $\mathrm{v}^{\prime \prime}=0$ and $\mathrm{v}^{\prime}=0$. When the vibrational frequency $v$ is identical in $\mathrm{S}_{0}$ and $\mathrm{S}_{1}$ the excitation energy for the most probable $\mathrm{S}_{0} \rightarrow \mathrm{S}_{1}$ transition, $\Delta E_{\max }$ (bold arrow), corresponds to $D_{\mathrm{o}}$ (the energy difference between the minima of the two potential energy surfaces). Thus, the difference $\Delta E_{\max }-\Delta E_{\mathrm{v}}$ depends on the zero-point vibrational energy, $\mathrm{ZPVE}=1 / 2 h v$, and the difference between $r_{\mathrm{eq}}{ }^{\prime}$ and $r_{\mathrm{eq}}{ }^{\prime \prime}$ in reduced coordinates, $d=$ $a^{1 / 2} r_{\mathrm{eq}}{ }^{\prime \prime}-a^{1 / 2} r_{\mathrm{eq}}{ }^{\prime}$ (with $a=4 \pi^{2} \mu v / h ; h$ the Planck constant and $\mu$ the reduced mass of the vibration). 